International Journal of Pure and Applied Mathematics

Volume 85 No. 5 2013, 859-868

ISSN: $1311-8080$ (printed version); ISSN: 1314-3395 (on-line version)

url: http://www.ijpam.eu

doi: http://dx.doi.org/10.12732/ijpam.v85i5.4

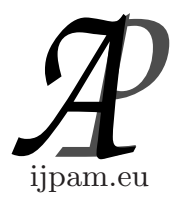

\title{
COUNTING WITH THE FINGERS
}

\author{
Yutaka Nishiyama \\ Department of Business Information \\ Faculty of Information Management \\ Osaka University of Economics
}

2, Osumi Higashiyodogawa Osaka, 533-8533, JAPAN

\begin{abstract}
It has been written that across the world there are 27 types of counting method using the fingers. For example, there are various methods for starting to count 'one' using either the index finger, thumb or little finger. In ancient Rome, people counted 'one' by bending their left little finger. Counting using the fingers differs according to region, ethnicity, and historical period. This chapter also discusses the 'evolutionary theory' of counting methods.
\end{abstract}

AMS Subject Classification: 01A02, 00A09, 97A20

Key Words: finger counting, counting with the fingers

\section{French People Start with the Thumb}

It was the summer of 2000. On my first family trip to Europe I had something of a cross-cultural experience. After visiting the Sacre Coeur Basilica on the Montmartre butte in the centre of Paris I was hungry, and spotting an open-air restaurant, something that looked like a crepe sprinkled with sugar caught my eye. At the restaurant I said, 'One of these please' in English but they didn't understand me. So then I said 'this', pointed at the food, and held up my index

Received: January 17, 2013

(c) 2013 Academic Publications, Ltd. url: www.acadpubl.eu 
finger to indicate ' 1 '. Once again they didn't understand. After another two or three attempts in silent dialog, I realized that while Japanese people indicate 1 using the index finger turned away toward the other person, French people use their thumb. Now that I mention it, I remember it was like a hitchhiker using their thumb to flag down a car, which felt a bit strange. Why is it that people who are born on the same Earth use different counting methods according to their country or region? This question has not left my head ever since.

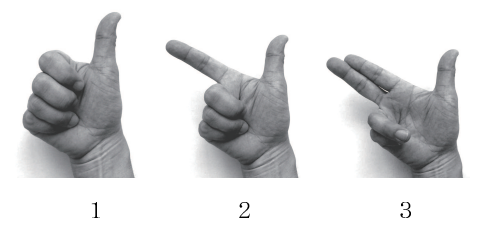

Figure 1: Counting with the thumb (Germany and France)

\section{Is Counting with Bent Fingers Inherent to Japan?}

When I was working as a member of the international exchange committee at university, I talked about this issue with a Swedish researcher, who told me that they definitely start counting from the thumb in Northern Europe. What's more, Europeans begin counting with the hand closed, i.e. with the 'scissors, paper, stone' symbol for 'stone', while Japanese people start from an open hand, i.e., with the symbol for 'paper'. We Japanese have a tradition of beginning to count with 'paper', and counting "one, two, threec" by bending down first the thumb and then each finger one after another. This goes as far as having the phrase yubiori kazoeru (finger-folding counting) in Japanese, so one can believe that this counting method has been used in Japan for a very long time. The Swedish researcher suggested that since Sweden, being in Northern Europe, is cold, people always keep their hands closed, while Japan is a warm country so people always keep their hands open. This means that the weather and climate is related to the way people count. This is an interesting idea. I wonder if this is really true.

This counting method also has a touch of a sad story associated with it. The story happened in India during the Second World War. An Indian girl had to introduce an Englishman who had called at her home to some Asian female friends. The problem was that the women were Japanese, and if this were 
known, she would be arrested in an instant. So she concealed their nationality and said that they were Chinese. The Englishman harbored some doubt, and he called upon them to 'try counting with your fingers, up to five'. They say the Englishman saw through the fact that they were Japanese by the way they counted? starting with their hands open, and bending their thumb and fingers in one by one (see Barrow, 2003).[1]

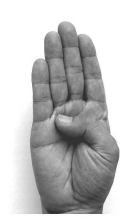

1

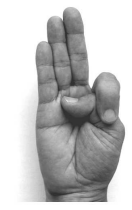

2

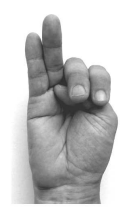

3

Figure 2: Bending the fingers beginning with the thumb (Japanese)

\section{Chinese People who Count to 10 on One Hand}

I once visited a university in China regarding an exchange program. Mentioning the topic of counting methods at a social reception, I was told that, "Chinese people and Japanese people count in the same way from the index finger. However, we count to 10 on one hand", and I was taught this counting method. 1 to 5 are counted in the same way as in Japan, but 6 is made by extending the thumb and little finger from the 'stone' position. It looks like the gesture for a telephone. In Hawaii people say "Hang loose!" and make this gesture as a greeting. Apparently this means "let's take it easy". Perhaps in the Chinese gesture, the thumb represents 5 and the little finger represents 1 , and these combine to make 6 .

7 is represented by extending the triple of the thumb, the index finger and the middle finger. Perhaps the thumb represents 5, and the other fingers combine to make the remaining 2. 8 is represented by using the thumb and index finger, and opening them to form the Chinese character for 8, ". 9 is represented by making the shape of the Chinese character for 9 , "with the index finger. In Japan this gesture means 'thief' so it feels a little strange to us. 10 is represented by crossing the index finger and the middle finger. This forms the Chinese character for 10, '? There is also a gesture in which the index fingers of both hands are used to form this cross shape, and the hand may be 
closed into a 'stone' again to represent 10, or both hands may be opened.

In Taiwan, 6 is the same as in China, but 7 to 9 are different. Also, I thought that it was only in China that they counted up to 10 on one hand, but the Masai tribe in Africa expresses the numbers up to 10 on one hand, and photographs of these hand symbols have been published. The Masai counting method differs from that of China (see Barrow, 2003 and Zaslavsky, 1999).[1] $[5]$

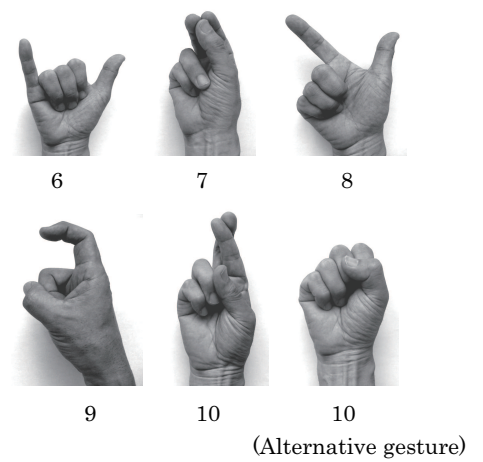

Figure 3: The Chinese gestures for counting 6 to 10

\section{The Distribution of Counting Methods on a World Map}

When counting with the fingers to communicate numbers to others, we Japanese begin counting from 1 with the index finger held up away from us, but French and German people begin with the thumb. I wanted to know the reason why we ended up different in this way. I was fortunate enough to be granted an opportunity for a year of overseas education in the United Kingdom in 2005. There were foreign students from 40 different countries from around world at Saint Edmund's College in Cambridge. Taking my lunch in the college dining hall, I made conversation regarding counting methods.

It seems that in the German they also start counting from the thumb, and they do so across the whole of Northern Europe. When starting with the thumb, I found that making a 3 was a bit tight. I asked a German about this and he demonstrated, smoothly opening his fingers, 'Ein, Zwei, Drei'. Then he said that making the Japanese 3 requires some force and is awkward. Hearing this, I noticed that our 3 is indeed difficult to form. We practice from when we are 
small, and come to think of this movement as perfectly natural. It seems that it is one of those things where it is impossible to say which method is easier.

A Filipino student taking part in this conversation mentioned that they start from the little finger. Since I was thinking that 1 would be made using the thumb or the index finger, this was quite unexpected. In Japan, extending the little finger means 'woman', so this felt a little strange to me. I understood from the literature that there are quite a few countries and regions where counting begins with the little finger (see Barrow, 2003 and Seidenberg, 1960).[1] [4] Again, asking someone from Bangladesh, I was told, 'This is how we make numbers and count', and the thumb was placed on the first joint of the index finger and moved in order through the joints of the fingers. 1 is made with the finger at the first joint, 2 at the second joint, and 3 at the third. Then they counted up to 10 on one hand. This method is also used in India, where the use of the joints of the index finger or the little finger to represent 1 varies by region, but they share the characteristic of using the joints of the finger. When I was in elementary school, we made a game of trying to correctly state the day on which someone was born based purely on their date of birth. At that time, we adopted a method using the finger joints. Perhaps this calculation method had its roots in India or Pakistan.

I have heard an explanation for the reason why Indians can perform this meticulous method of counting, namely, that their thumbs have three joints. Do you really think so? In relation to this, 'The History of Numbers' (p22) contains a diagram of a triple-jointed thumb (see Donny, 1998).[2] This concerns a finger arithmetic method illustrated in the 8th century by the English monk, the Venerable Bede. Perhaps there really is a group of people whose thumbs are triple jointed.

As we have seen above, there are many methods for counting, no matter which digit is used for 1 . Wouldn't it be wonderful to have a color coding scheme for countries and regions? It occurred to me that with such a graph, one might be able to understand the history of the development of these methods. I was very fortunate to find an excellent reference. Seidenberg performed an investigation in 1960, which reached every corner of the world, and published his findings (Seidenberg, 1960).[4] He classified every method according to whether it starts on the left little finger, the right little finger, the index finger, the thumb or a finger joint, and drew up a world map. This map is introduced in 'Pi in the Sky' (see Barrow, 2003).[1] According to the results it might be appropriate to call Africa the origin of humanity, because all of the counting methods exist there. Japan is classified as starting from the thumb in this work. Such a survey requires extensive time and manpower, and if this investigation 
were performed again, over 40 years later when the internet is prevalent, even more detailed results could no doubt be assembled.

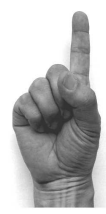

1

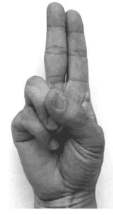

2

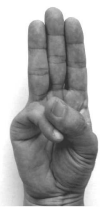

3

Figure 4: Counting using the index finger (China and Japan)

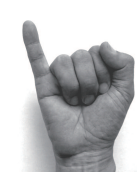

1

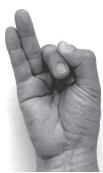

2

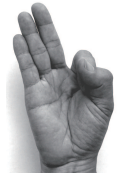

3

Figure 5: Counting using the little finger (Philippines)

\section{In Ancient Rome they Began with the Left Little Finger}

Now we know that there are differences according to county and region, such as using the little finger, the index finger, the thumb or the finger joints and so on, but this is the way things are today. How did people in ancient times count, and which finger did they start from?

Menniger's 'A Cultural History of Numbers' (2001) contains the following explanation. [3] Counting on the fingers was for the most part handed down by oral tradition. Definitive documents such as textbooks have not been left behind through the time since the Roman era, and the first recorded method was made by the English Benedictine monk, the Venerable Bede. He died in $735 \mathrm{AD}$, but left behind a document entitled, 'Arithmetic and Conversation using the Fingers'. In this document 1 is expressed by bending the left little finger into the palm of the hand. 2 is expressed by bending the ring finger down beside it, and 3 by bending the middle finger down beside that. 


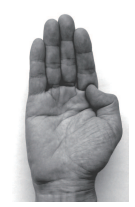

1

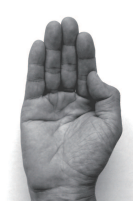

2

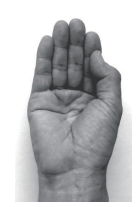

3

Figure 6: Counting using the joints of the finger (India, Pakistan and Bangladesh)

Another counting method using the fingers was illustrated in a document entitled 'The Arithmetic System', published in Venice in 1494, and written by the Italian mathematician Luca Pacioli. This also expressed 1 by folding down the left little finger. This indicates that from ancient times up until the middle ages, when people counted on their hands, they began in general with the left little finger. This fact seems particularly unusual to me, as I was thinking that counting from 1 would begin with the thumb or the index finger, and that even if it began with the little finger, I thought it would involve opening the fingers. I wonder why they used the left hand? Also, why did they go from the little finger? Also, another question emerges. I found the method which involves bending the left little finger hard to perform. In West Africa, bending the little finger on the left hand is indeed a 1, but there is a diagram in Zaslavsky's book of illustrations which explains pictorially that in this case the index finger of the right hand is used to push down the left middle finger (Zaslavsky, 1999).[5] Perhaps it is difficult to bend it by itself after all? Or perhaps people in ancient times learned to bend it through practice.

I have previously discussed the fact that computers are based on binary. I have heard it explained that if we used this binary system with both hands, we could count to 1024. If an extended finger represents 0 (zero), and a bent finger represents 1 (one), then each finger represents one bit, and since we have 10 fingers we can express a 10 bit number. Since $2^{10}=1024$, it was explained that we could count up to 1024, but this is truly the kind of topic you'd expect a computer scientist to enjoy, and it's doubtful whether we could really manage this kind of finger bending. The Venerable Bede and Luca Pacioli both explained and illustrated how to count as far as 10,000 using both hands. This is well beyond 1024. In addition, they didn't just explain how to count, but also how to perform multiplications and so on with the fingers, so we can 
say that this was indeed a technically well-developed field.

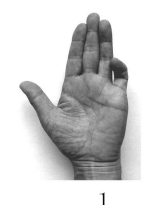

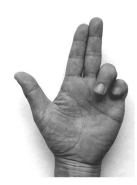

2

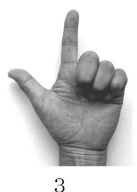

Figure 7: Counting using the little finger of the left hand (ancient Rome)

\section{Food Culture, Lifestyle and Counting?}

Searching the internet, it was written that across the world there are 27 types of counting method using the fingers. Wouldn't there be even more considering the possible combinations? Counting using the fingers differs according to region, ethnicity, and historical period. But why did the methods change in these ways, and eventually arrive at the present situation? It's fun to think about this 'evolutionary theory' of counting methods from various angles.

To begin with, the ancestors of humanity, the Africans, possess all of the counting methods. Expressing 1 with the thumb, with the index finger, the right little finger, the left little finger, etc., all of the archetypes exist there. There are also many tribes such as the Masai who count from 1 to 10 using one hand, and counting systems can be said to be considerably advanced. Also, of particular note, not only base 10, but also counting methods and numerical notation systems using the principle of binary exist there. Do these complex ways of presenting the fingers according to binary patterns constitute evidence of an ability to move the fingers freely? There are also tribes that have adopted base 20. Perhaps this owes to the fact that a barefoot lifestyle makes the toes available for use too. This topic is dealt with in more detail in the book by Zaslavsky (see Zaslavsky, 1999).[5]

We Japanese do not use binary in the same way as these African peoples. In that regard, counting methods may be considered to have developed so that we don't only rely on the fingers, but use other methods such as calculators, etc. It's also possible to imagine that the reason we don't use base 20 is that because we don't have bare feet. By starting to wear shoes or other such footwear that hides the toes away, we settled on base 10, which uses the hands alone.

In ancient Rome and in the middle ages, bending the left little finger was 
1. Maybe the reason for using the left hand was that the right hand was used for more important things. Also, bending the little finger is difficult, and yet we don't know the reason for its use. As time advanced, people began to communicate 1 using the index finger in China and Japan, using the thumb in Germany and France, and using the little finger in the Philippines, etc. Counting methods are a part of our national and ethnic identity. Language, religion, currency, systems of measurement, and so on are characteristics of our ethnicity, and just as other differences express differentiation among peoples. Perhaps a conscious awareness of these differences led to the differentiation of counting methods.

Maybe the difference between starting from the open 'paper' condition and proceeding by bending the fingers, versus starting from the closed 'stone' condition and extending the fingers, is related to the air temperature. Perhaps the natural position of the hand is either 'paper' or 'stone' for different races. In hot countries it would be 'paper' and in cold countries it would be 'stone'. According to such thinking, Africa, being a hot country, would count from 'paper', while the tribes who moved to colder Northern countries would count from 'stone'.

While conversing about the different counting methods with the foreign exchange students in the college dining hall, the following thought suddenly crossed my mind. Perhaps the ways of counting with the fingers are related to food culture. Meals in Europe involve a knife and fork. When cutting meat it is necessary to firmly grasp the knife and fork. When so doing, it is the thumb which is free to move, so is this why extending the thumb came to represent 1? Also, the countries in Northern Europe are cold, so many people keep their hands closed and perhaps the easily extended thumb naturally came to represent 1.

Once a method of counting with the fingers is established, the other fingers begin to take on other meanings. In the West, the thumb represents 1 , but in Japan it indicates 'man'. In the Philippines, the little finger represents 1, but in Japan it indicates 'woman'. The Chinese 9 indicates 'thief' in Japan. On the other hand, the Japanese 2 is the 'victory' sign in the United Kingdom. It is common for the meanings of the fingers to be completely different in different countries. The English zoologist Desmond Morris researches this in his book, 'Bodytalk: A World Guide to Gestures' (Sanseido Co., Ltd., translated by Toyama Yasuko). Interested readers should refer to the references. Counting methods using the fingers are not limited to the expression of numbers, they appear to go as far as influencing each county's culture. 


\section{References}

[1] J.D. Barrow, (Transl. M. Hayashi), Tenku no Pi [Pi in the Sky: Counting, Thinking, and Being], Tokyo: Misuzu Shobo (2003).

[2] D. Guedj, (Transl. I. Nanjyo), Su no Rekishi [L'empire des nombres], Osaka: Sogensha (1998).

[3] K. Menniger, (Transl. M. Uchibayashi), Zusetsu Su no Bunkashi [Number Words and Number Symbols: A Cultural History of Numbers], Tokyo, Yasaka Shobo (2001).

[4] A. Seidenberg, The Diffusion of Counting Practices, Univ. of California Press (1960).

[5] C. Zaslavsky, Africa Counts: Number and Pattern in African Culture, Lawrence Hill Books (1999). 